\title{
Traditional Education
}

National Cancer Institute

\section{Source}

National Cancer Institute. Traditional Education. NCI Thesaurus. Code C159512.

Education that takes place in a public school, divided into grades, and governed by a school district. 